\title{
Arthroscopy in the treatment of knee septic arthritis
}

\author{
Mahmood A. Al-Jumaily * Ahmad I. Al- Jibbory **. \\ *Department of surgery, Mosul College of Medicine, University of Mosul; ** Department of Orthopedics, \\ AL-Jamhory Teaching Hospital, Mosul.
}

(Ann. Coll. Med. Mosul 2007; 33(1\&2):20-25)

Received: $7^{\text {th }}$ Aug 2006; Accepted: $3^{\text {rd }}$ Jul 2007

\section{ABSTRACT}

Objective: to evaluate the use of arthroscopic irrigation in the treatment of septic arthritis of the knee joint.

Methods: This case series study. Was conducted in Al-Jamhori Teaching Hospital in Mosul, from August 2003 to December 2005, on twenty patients with septic arthritis of knee joint who presented with pain, fever, swelling and limitation of movements of their affected knee joint. The mean age of the patients was 31.5 years, and the female to male ratio was 1:1.5. Arthroscopic irrigation was arranged within 24 hours of admission, suction drain used, with the use of intravenous antibiotic for 7 days then change to oral antibiotic for 5-6 weeks. laboratory investigation and X-ray were done, aspiration of synovial fluid was send for analysis, direct gram stain, culture and sensitivity.

Results: Synovial biopsy confirmed the diagnosis and showed typical changes of septic arthritis. We achieved $60 \%$ excellent, $30 \%$ good and $10 \%$ acceptable results of the knee joint depending on clinical evaluation and laboratory investigation. Most of patients were delayed in presentation. Staphylococcus aureus was the commonest causative organism.

Conclusion: Arthroscopic irrigation of knee joint has encouraging results in the treatment of septic knee with low incidence of complications.

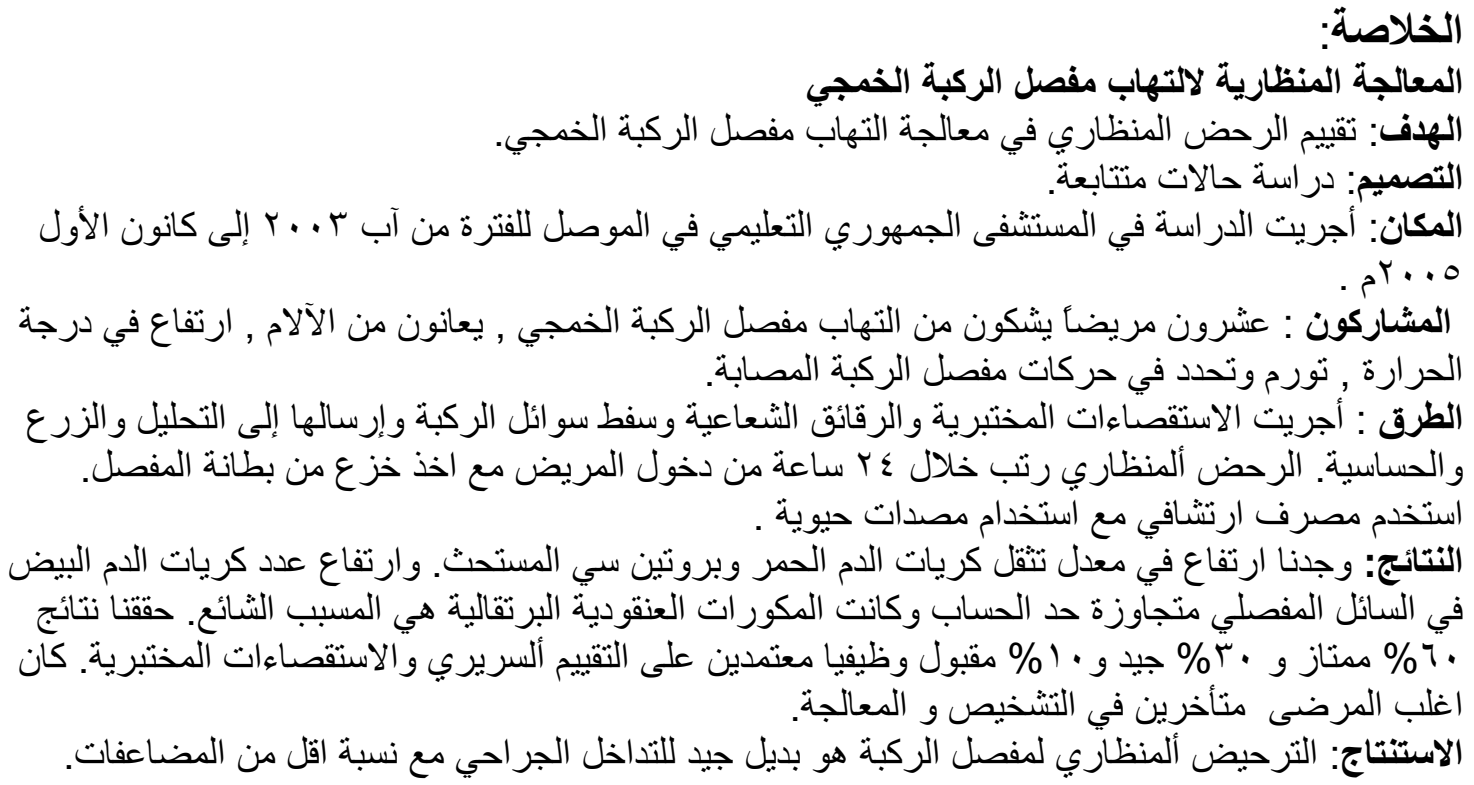


$\mathrm{S}$ eptic arthritis is an orthopedic emergency that can lead to rapid joint destruction and irreversible loss of function with both significant morbidity and mortality which result from bacterial invasion of joint space ${ }^{1-9}$. The most commonly involved joint is the knee $(50 \%$ of cases) ${ }^{10}$. The major consequence of bacterial invasion is damage to articular cartilage. These destructive processes are well advanced as early as 3 days into the course of untreated infection ${ }^{10}$.

Septic arthritis of the knee joint may present with pain, fever, swelling, tenderness, warmth, effusion, and impaired joint movement ${ }^{11}$. Arthroscopic lavage has been used increasingly in the management of septic arthritis. The advantages are that extensive debridement can be performed with a small incision and there is a more rapid recovery period and the patient needs short term hospitalization ${ }^{12}$. With early onset of therapy, arthroscopic treatment leads to an effective resolution of infection with better functional results than open arthrotomy ${ }^{13,14}$

The aim of this study is to evaluate the use of arthroscopic irrigation in the treatment of septic arthritis of the knee joint and to present pattern of septic arthritis of the knee joint in our community.

\section{Patients and methods}

Twenty patients with septic arthritis of the knee joint were treated by arthroscopic treatment. The study was carried out in AlJamhori Teaching Hospital in Mosul between August 2003 and December 2005. Full history of illness was taken and detailed clinical examination performed. Aspiration of the joint and synovial fluid analysis including culture and sensitivity, serological examination, WBC count, differential count, ESR, CRP, and radiographs were done. All patients received broads spectrum antibiotics intravenously in divided dose after aspiration until the result of culture and sensitivity was obtained.

Arthroscopy was done in operating room under general anesthesia through anterolateral approach. Examination of the knee begins first. During the procedure we use the high flow irrigation using (5) liter of ringer lactate and suction sheath connected days after arthroscopic treatment, passive movements were allowed. After two months of follow up, three patients had restriction of to negative pressure sucker. The high flow irrigation is used to distend the joint, to clear any debris, to release adhesions, and to clear the joint from the toxic material produced by bacteria and polymorphonuclear leukocytes. Biopsy taken from the synovium, suction drain left, and dressing with Robert- Jones bandage. Parenteral analgesic and intravenous antibiotics were given to the patients postoperatively, and patients encouraged doing quadriceps exercises. The suction drain being inspected and also the amount of the fluid collected. Mobilization of the patient out of bed on crutches was allowed as soon as the circumstances of the patient permitted. At the $3^{\text {rd }}$ day, the wound was inspected and the suction drain removed and more active exercises allowed. Full weight bearing was allowed (14) days after the operation and stronger active exercise encouraged. The follow up regimen continued every week for (8 weeks) or until the patient is completely normal. The cured patients were advised to visit us every (8 weeks) for physical and laboratory evaluation. The last outcome results were classified to four grades according to the last clinical examination ${ }^{14}$ : "Excellent" normal movements, no pain, no swelling and no instability (resolution): "Good" no pain, few degrees of limitation in movements, no swelling and no instability. "Accepted": occasional pain, some limitation of movements, 10-20 degrees, stable joint and mild swelling; "Poor": painful joint, marked stiffness, persisted swelling; and instability.

\section{Results}

The female to male ratio was 1:1.5 (8 females and 12 males). The mean age of the affected cases was (31.5) years ranging from $3-65$ years. Pain, swelling and restriction of movements of the knee joint were present in all cases (100\%), (fig.1). Immediate gradual decline of pain was present on all cases after treatment $(100 \%)$, and most of the patients had complete relief of pain after two weeks of treatment (16 cases $80 \%$ ). Four patients had pain on extremes of movement (20\%). Swelling markedly decreased 3 days after operation, when the dressing was removed, but two patients still had mild swelling (10\%). Four movement between (30 - 50 degrees), but no bony ankylosis. Fever was present in $70 \%$ of patients (14 cases).Two days after 
treatment, the temperature declined in all patients and became afebrile after 4 days, (fig.1). The mean hospital stay was five days.

The ESR was elevated in all cases, ranging from $60-130 \mathrm{~mm} / \mathrm{hr}$, with a mean of $73.4 \mathrm{~mm} / \mathrm{hr}$, (fig.2). It started to decline gradually, and returned to normal level six weeks following arthroscopic treatment in 18 cases $(90 \%)$, but remained high in two (10\%),( fig. 2). The CRP was elevated in all cases with a value ranging from $76-150$ $\mathrm{mg} / \mathrm{l}$, with a mean value of $107.4 \mathrm{mg} / \mathrm{l}$, (fig.1). After arthroscopic treatment, CRP showed rapid decrease, except in two cases (10\%), (Fig. 3). White blood cell count was increased in $60 \%$ of patients (12 cases), (fig. 1 ).

Under full aseptic technique aspiration of the knee joint was done, the aspirates sent for synovial fluid analysis, direct gram stain, Ziehl- Neelsen stain for AFB, and for culture and sensitivity. Cells were mainly neutrophils (95\%). Viscosity was decreased. Protein was high in variable values, and the sugar was decreased in swelling (10\%). Four comparison to random blood sugar in $60 \%$ of cases (12 cases). Gram stain was positive in three cases (15\%). AFB was negative in all cases. Culture was positive in 17 patients (85\%). The most common microorganism was Staph, aureus, 11 (55\%) of patients. Three cases (15\%) showed no growth probably due to receiving antibiotics.

Histopathological examination was carried out for synovial biopsy in the laboratory of Al- Jamhori Teaching Hospital in Mosul which confirmed the diagnoses of septic arthritis in all 20 patients. All histopathological examinations showed inflamed synovium with acute inflammatory cell infiltration (mainly neutrophils). Periarticular soft tissue swelling in plain radiographs was the most common finding. We found two cases of rheumatoid arthritis complicated by septic arthritis. Another three cases had history of joint injections within one week .One case underwent operation in the knee joint ( menisectomy) complicated by septic arthritis four weeks after operation. Depending on functional and clinical assessment arthroscopic lavage achieved $60 \%$ excellent results, $30 \%$ good results and $10 \%$ accepted results.

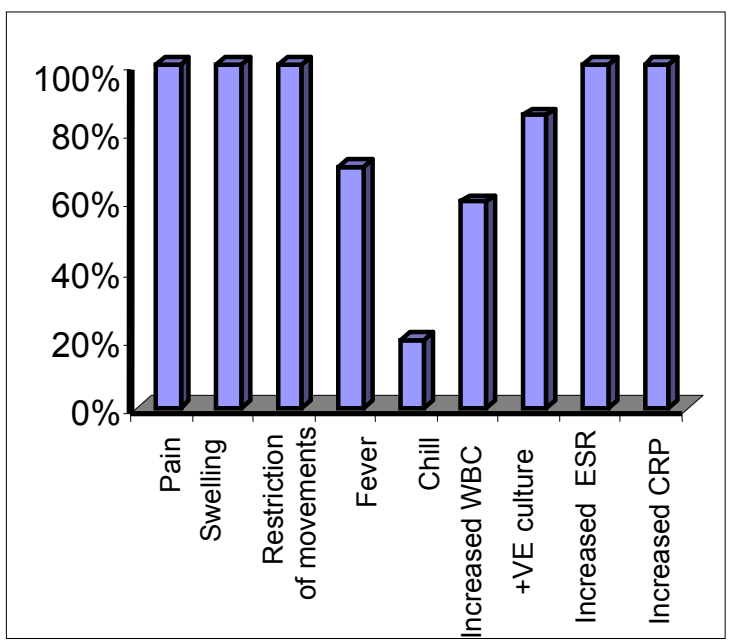

Fig (1): Frequency of main clinical features and the investigations of patients.

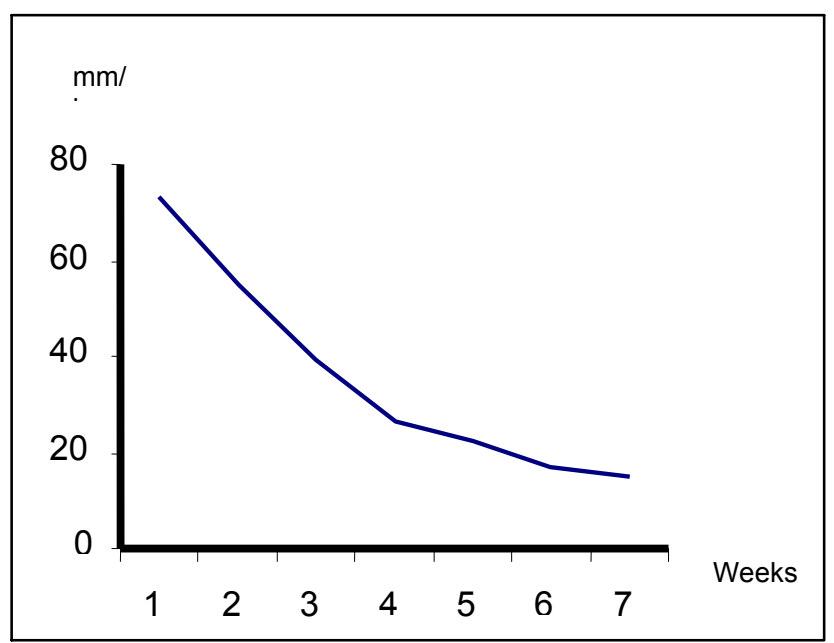

Fig (2): Mean value of ESR of patients at time of presentation and weekly decrease on follow-up post operatively. 


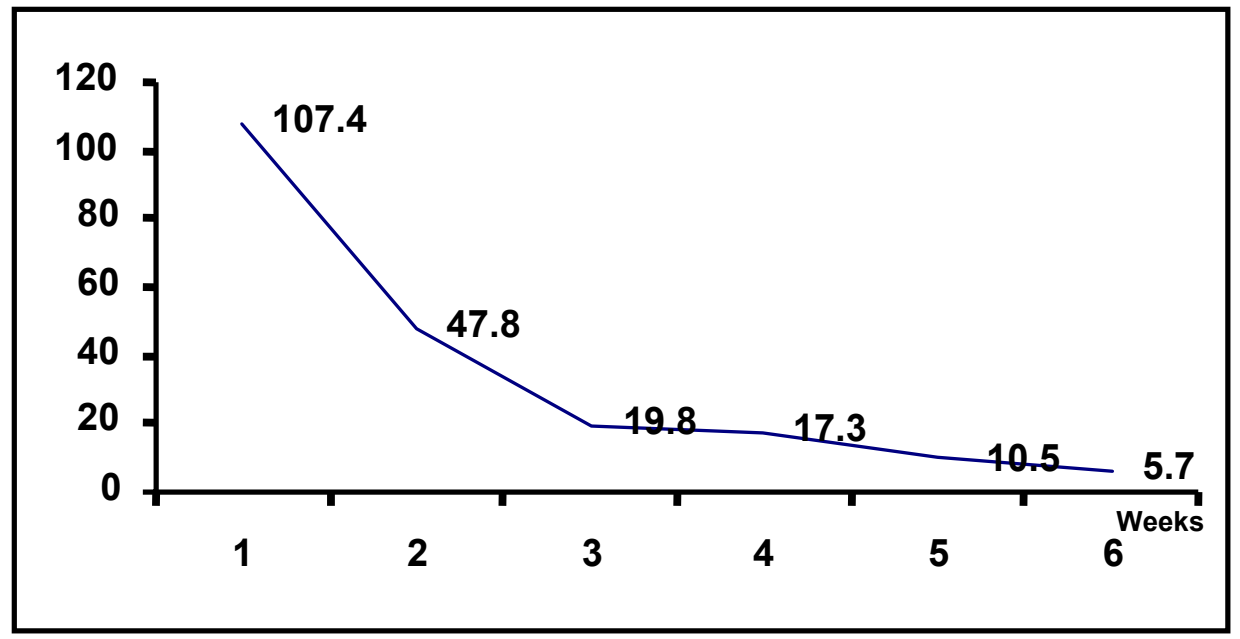

Fig (3): Decline in the mean value of CRP of patient followed up weekly.

\section{Discussion}

Acute septic arthritic is an acute inflammation, caused by pus forming microorganism $^{(15)}$. Despite improvement in antibiotic therapy of septic arthritis mortality and morbidity have not improved significantly over the last two decades $^{(16,17)}$. All the patients studied had the clinical diagnosis of acute septic arthritis of the knee joint confirmed by bacteriologic and histopathological investigations. In our study the occurrence of the disease was higher in males than females; similar result was reported by Chen. et. al and Youssif ${ }^{(18,19)}$.

Pain was the first and most frequent presenting symptom (100\%) and was present on movement and rest. In the study of Gupta, pain was present in $85 \%$ of his cases 1 . Fever; was present in $70 \%$ of patients, in all of them their temperature started to decline at the second day after arthroscopic treatment. The fever was described in only $60 \%$ in Brusch study and $80 \%$ in Shahbaz study ${ }^{(10-20)}$. They attributed it to the incorrect use of antibiotics prior to the diagnosis, on trial and error bases without proper culture and sensitivity.

Swelling was present in all cases and decreased markedly three days after operation in $90 \%$ of cases. In Gupta study swelling was present in $77 \%$ of his cases $^{(1)}$. Limitation of movement was present in all cases. After two month of follow up three patients $(15 \%)$ had some restriction of movement, two of them already had rheumatoid arthritis. Chen $\mathrm{CH}$ reported in his study that the common presenting features were pain, swelling and restriction of movements ${ }^{(18)}$.

In our study the pre intervention period was $7-20$ days. In study by Shabir, the pre intervention period was $5-13$ days ${ }^{(15)}$. Our study indicates that most of the patients presented late on the course of the disease; this may be attributed to vague symptoms in the initial stage, or the patients were improperly treated by general practitioners.

White blood cell count was increased in $60 \%$ of cases. Gupta reported increase in WBC count in $63 \%$ of his cases ${ }^{(1)}$, Shabir in his study the WBC count was increased in $65 \%{ }^{(15)}$. ESR and CRP were increased in all cases (100\%). Shabir in his study the ESR was elevated in $72 \%$ of patients ${ }^{(15)}$, and similar results were achieved by Chen et. $\mathrm{al}^{(18)}$. Gupta et al in their study, the ESR and CRP were elevated in all cases $100 \%{ }^{(1)}$. In Weston study the ESR was moderately increased and occasionally normal; in contrast the CRP which was usually raised and greater than $100 \mathrm{mg} / \mathrm{I}^{(2)}$. Jerosch, in his study the CRP was the most sensitive parameter ${ }^{(21)}$.

The culture of synovial fluid was positive in $85 \%$ of patients and our bacteriological results are similar to those reported by Le - Dante et. al ${ }^{(15-17)}$, Youssef and York ${ }^{(19)}$ and Dagan ${ }^{(22)}$, who found staphylococcus aureus the commonest organism. We 
have confirmed that staphylococcus aureus was the most common bacteria responsible for acute septic arthritis (55\% of cases). Weston et al in his study, synovial fluid culture was positive in $67 \%$ with the commonest microorganism being staphylococcus aureus ${ }^{(2)}$. A study, of Gupta demonstrated that staphylococcus aureus was the commonest pathogen in $71 \%$ of cases $^{(1)}$. Gordian, in his study the synovial fluid culture was positive in $78 \%$ of cases and the staphylococcus aureus was the most common causative organism $42 \%{ }^{(23)}$. Smith, in his study, staphylococcus aureus was the commonest causative organism $66 \%{ }^{(24)}$. Kaandrop et al in his studies, gave broadly similar result for the spectrum of organisms seen ${ }^{(4)}$. The incidence of gonococal arthritis is much lower than in UK survey and in accordance with studies from Europe ${ }^{3,4}$. This continues to be in contrast with reports from urban area of north America and the tropical Australia ${ }^{(2-5)}$.

We have found that $30 \%$ of septic arthritis of the knee joint had underlying causes; joint injection (15\%), rheumatoid disease $(10 \%)$ and previous surgery $5 \%$. In other prospective hospital - based study, the incidence of underlying joint disease was $60 \%{ }^{(1-16)}$. Retrospective analysis may not have the opportunity to evaluate all symptoms and investigation, and may therefore underestimate preexisting joint disease ${ }^{(2,3-5-17)}$. In another study achieved by Weston, previous joint disease was evident in $35 \%$ (RA was $16 \%$ ) compared with expected frequency in population $1 \%{ }^{(2)}$.

Our study demonstrates that septic arthritis of the knee joint is associated with $15 \%$ morbidity with no mortality. In other studies the mortality rate was about 10$15 \%$, and the morbidity was about 30 $50 \%,(2,3,7,16,20,23,26,27)$. Most of the mortality was seen in the elderly, those with bactraemia, and those with poly- articular disease. The absence of mortality in our patients might be related to the fact that we don't deal with immune compromised patients and patients at age extremes. The association between delay in diagnosis and increased morbidity has been noted before ${ }^{(2,3)}$. It emphasizes the need to maintain a high index of suspicion of the condition, particularly in at risk groups. In our study, the mean hospital stay was five days while in the study by Weston, the mean hospital stay was 26 days $^{(2),}$ and the study of Gupta, the median in -patient stay was 25 days $^{(1)}$. Smith, in his study, the average hospital stay was 17 days $^{(24)}$.

Depending on clinical evaluation, we achieved $60 \%$ excellent result, $30 \%$ good and $10 \%$ accepted via arthroscopic treatment. Jerosch, in his study of 22 septic arthritis treated by arthroscopy, he achieved $100 \%$ excellent and good results ${ }^{(21)}$. Smith achieved $93 \%$ excellent and $7 \%$ good result, no poor results, recurrence or osteomyelitis ${ }^{(24)}$. Vispo Seara achieved in his study $61 \%$ excellent, satisfactory in $20 \%$ and poor results in $19 \%{ }^{(28)}$. Gordian reported $91 \%$ cure rate, $4 \%$ need open arthrotomy revision ${ }^{(23)}$. Most of the authors stressed that the time elapsed between the onset of symptoms and the starting of treatment is an important prognostic factor, other factors included age of the patient, pre existing joint disease and type of microorganism ${ }^{(21-23-24-28)}$. Our result is encouraging though it looks inferior to the other studies; this might be due to the delay in management in our cases.

We can conclude that arthroscopic lavage of knee septic arthritis had encouraging results due to small incision, short hospital stay, visualization of the interior of the joint, release of adhesions, debridement, synovial biopsy taken and synovectomy can be done when needed, and early rehabilitation and less limitation of joint movement.

\section{References}

1- Gupta MN, Sturrock RD, Field M. Patients with adult-onset septic arthritis. A prospective communitybased study, Arthritis rheum 2001; 40:24 - 30 .

2- Weston VC, Jones AC, Bradbury N, Fawthrop F, Doherty M. Clinical features and outcome of septic arthritis in a single UK Health District 1982 - 1991. Ann Rheum Dis. 1999; 58:214-219.

3- Cooper C, Cawley MI. Bacterial arthritis in an English health district, a 10 year review, Ann Rheum Dis. 1986; 45:458-463. 
4- Kaandrop CJE, Dinant HJ, van de laar MAFJ, Bernelot Moens HJ, prins APA, Dijkamans BAC. Incidence and sources of native and prosthetic joint infection: a community based prospective survey. Ann Rheum Dis. 1997; 56: 470-475.

5- Morgan DS, Fisher D, Merianos A, Currie BJ. An 18 year clinical review of septic arthritis from tropical Australia. Epidemiol infect 1996; 117:423-428.

6- Peters RHJ, Rasker JJ, Jacobs JWG, Prevo RL, Karthaus RP. Bacterial arthritis in a district hospital. Clin Rheum 1992; 351-355.

7- Kaandrop CJE, Krijnin P, Bernelot Moens HJ, Habbema JDF, van Scaardenburg D. The out come of bacterial arthritis. A prospective community-based study Arthritis Rheum 1997; 40:884-892.

8- Ryan MJ, Karanagh R, Wall PG, Hazleman BL. Bacterial joint infections in England and Wales: analysis of bacterial isolates over a four year period. $\mathrm{Br} \mathrm{J}$ Rheumatol 1997; 36: 370-373.

9- Keith D. Williams. Infectious Arthritis, in: Canale S. Campbell's operative orthopaedics. $9^{\text {th }}$ edition., Mosby Pennsylvania; 1998: 601-602, 1455.

10- Brusch JL, Mileno DM. septic arthritis. E medicine. 2005; 18: 159 - 167.

11- Alexander R; Vaccaro. Orthopaedic Knowledge update. $8^{\text {th }}$ edition. American Academy of orthopaedic surgeons; 2005:222.

12- Parisien JS, Shaffer B: arthroscopic management of pyoarthrosis. Clin orthop 1992, 275:243-247.

13- Dlabach AJ. Infectious Arthritis Canale ST ;Campbell's operative orthopaedics. $10^{\text {th }}$ edition . Mosby. Pennsylvania; 2003: 692.

14- Wirtz D, Marth M, Miltner O, Schneider U. Septic arthritis of the knee in adults: treatment by arthroscopy or arthrotomy. International orthopaedics. 2001, (25): 239-241.

15- Shabir M-Acute septic arthritis and its management. Journal of post graduate medical institute. 2004, 18 :269-274.

16- Kaandrop CJE, van Schaardenburg D, krijnen $P$, Habbema JDF. Risk factor for septic arthritis in patients with joint disease a prospective study .Arthritis Rheum 1995; 38:1819-1825.

17- Le Dantec L, Maury F, Flipo RM et al .peripheral pyogenic arthritis. A study of one hundred and seventy nine cases .Rev Rheum Engl Ed 1996; 63: 103-110.

18- Chen $\mathrm{CH}$, Lee ZL, et al. Acute septic arthritis of the hip in children. Clinical analysis of 31 cases. Cheng Keng I Hush, 1993; 16 (4) : 239.

19- Youssef PP, York JR. septic arthritis a second decade of experience. Aust. N. ZJ Med, 1994; 24 (3): 307.

20- Shahbuz H, James W. Septic arthritis current treatment option in infections disease 2001; 3 : $279-286$.

21- Jerosch J, Prymk. M. Arthroscopic treatment of septic arthritis. Surgical technique and results. Der unfallchirng 1998; 6:454-460.

22- Dagan R. Management of acute haemotogenous Osteomyelitis and septic arthritis in pediatric patients. Paediat infect Dis J 1993; 12:88.

23- Gordian S, Maokus S. Kuster, Frank kleinstuck, andre gachter. Arthroscopic management of septic arthritis: stage of infection and results. Knee surgery, spots traumatology, arthroscopic 2000;5:270 - 274.

24- Smith MJ. Arthrocopic treatment of the septic knee. Arthroscopy 1986; 2 : 3034.

25- Goldenberg DL. Septic arthritis. Lancet 1998; 351: 197 - 202.

26- Nalini R. septic arthritis. Current treatment options in infections diseases. 2002,4:279-287.

27- Dubost JJ, Fis I, Denis P, et al. Poly articular septic arthritis. Medicine 1993; 72: 296 - 310.

28- Vispo Seara J , Barthel T, Schmitz H, Eulert J. Arthroscopic treatment of septic joints: prognostic factors. Archives of orthpaedic and Trauma surgery 2002; 4: 204 - 211. 